\title{
PREPARAÇÃO DE VIDROS E VITROCERÂMICAS DE ÓXIDOS DE METAIS PESADOS CONTENDO PRATA: PROPRIEDADES ÓPTICAS, ESTRUTURAIS E ELETROQUÍMICAS
}

\author{
Bruna A. Bregadiolli \\ Departamento de Física, Faculdade de Ciências, Universidade Estadual Paulista, Av. Eng. Luiz Edmundo Carrijo Coube, 14-01, \\ 17033-360 Bauru - SP, Brasil \\ Ernesto R. Souza e Fernando A. Sigoli \\ Instituto de Química, Universidade Estadual de Campinas, Cidade Universitária Zeferino Vaz, CP 6154, 13083-970 Campinas - \\ SP, Brasil \\ José. M. A. Caiut \\ Departamento de Química, Faculdade de Filosofia Ciências e Letras de Ribeirão Preto, Universidade de São Paulo, Av. Bandeirantes, \\ 3900, 14040-901 Ribeirão Preto - SP, Brasil \\ Mônica A. S. Alencar e Assis V. Benedetti \\ Instituto de Química, Universidade Estadual Paulista, CP 355, 14801-970 Araraquara - SP, Brasil \\ Marcelo Nalin* \\ Departamento de Química, Universidade Federal de São Carlos, CP 676, 13565-970 São Carlos - SP, Brasil
}

Recebido em 13/7/11; aceito em 31/10/11; publicado na web em 2/3/12

\begin{abstract}
PREPARATION OF GLASSES AND GLASS CERAMICS OF HEAVY METAL OXIDES CONTAINING SILVER: OPTICAL, STRUCTURAL AND ELECTROCHEMICAL PROPERTIES. Silver containing heavy metal oxide glasses and glass ceramics of the system $\mathrm{WO}_{3}-\mathrm{SbPO}_{4}-\mathrm{PbO}-\mathrm{AgCl}$ with different $\mathrm{AgCl}$ contents have been prepared and their thermal, structural and optical properties characterized. Glass ceramics containing metallic silver nanoparticles have been prepared by annealing glass samples at temperatures above the glass transition and analyzed by transmission electron microscopy and energy dispersive X-ray microanalysis. The presence of the metallic clusters has been also confirmed by the observation of a surface plasmon resonance band in the visible range. Cyclic voltammetric measurements indicated the presence of metallic silver into the glasses, even before to perform the thermal treatment.
\end{abstract}

Keywords: glass; glassceramics; nanoparticles.

\section{INTRODUÇÃO}

Vidros são materiais ideais para aplicações em fotônica, principalmente devido à facilidade de manipulação de suas propriedades químicas e físicas. As composições dos vidros podem ser formuladas dependendo do tipo de utilização desejada, por exemplo, vidros à base de fluoretos são empregados como amplificadores ópticos em telecomunicação ou na fabricação de lasers; vidros contendo calcogenetos são a base para a fabricação de dispositivos operando na região do infravermelho médio, os quais podem ser usados, por exemplo, como lentes de câmeras de visão noturna. Na família dos vidros óxidos existem duas classes principais de vidros com aplicações distintas: vidros à base de sílica $\left(\mathrm{SiO}_{2}\right)$ e vidros à base de óxidos de metais pesados (VOMP) tais como, $\mathrm{Sb}, \mathrm{Bi}, \mathrm{Pb}, \mathrm{W}, \mathrm{Ga}, \mathrm{Ge}, \mathrm{Te}$ etc. A primeira é sem dúvida a classe mais importante comercialmente e inclui aplicações que vão desde a preparação de vidros para laboratório até a utilização como fibras ópticas ou redes de Bragg. Entretanto, a segunda classe vem emergindo como um importante elo que permite diversas aplicações especiais, principalmente no campo da fotônica. Neste sentido, os VOMP surgem como materiais muito promissores e vários resultados interessantes vêm sendo relatados na literatura. ${ }^{1-3}$ Uma aplicação para os VOMP é a preparação de chaveadores ópticos, os quais requerem materiais com baixa perda linear e não linear, grande não linearidade Kerr e resposta ultrarrápida. ${ }^{2}$

Vale ressaltar que o papel da Química Inorgânica e o conhecimento das propriedades fundamentais dos elementos são determinantes para o desenvolvimento de novas composições vítreas com potencial

*e-mail: mnalin@ufscar.br para tais aplicações. Recentemente, com o advento da nanotecnologia surgiram novas aplicações para os VOMP. Um exemplo é o uso de matrizes vítreas contendo nanopartículas de metais nobres para aplicações tanto em óptica não linear como em amplificadores ópticos contendo terras raras..$^{4-9}$ Entretanto, a preparação de tais materiais não é trivial, uma vez que vários fatores devem ser levados em consideração e podem alterar as propriedades finais dos vidros. No que diz respeito à preparação de VOMP contendo nanopartículas e suas possíveis aplicações em óptica não linear, um dos pontos-chave é a obtenção de uma matriz vítrea que permita a incorporação dos metais (na forma de óxidos ou halogenetos) e que cristalize de forma homogênea. O segundo é o controle do crescimento das nanopartículas, que pode ser feito por meio de tratamento térmico controlado em temperaturas estabelecidas experimentalmente.

Neste trabalho foi estudada a preparação de VOMP no sistema $\mathrm{WO}_{3}-\mathrm{SbPO}_{4}-\mathrm{PbO}-\mathrm{AgCl}$ e foi realizado um estudo sistemático das propriedades ópticas, estruturais e eletroquímicas destes materiais em função da concentração de $\mathrm{AgCl}$, bem como da preparação de vitrocerâmicas transparentes contendo nanopartículas metálicas.

\section{PARTE EXPERIMENTAL}

\section{Síntese dos vidros}

A síntese dos vidros foi realizada usando os seguintes reagentes: $\mathrm{WO}_{3} 99 \%, \mathrm{PbO} 99 \%$ e $\mathrm{AgCl} 98 \%$ (Aldrich) e $\mathrm{SbPO}_{4}$ sintetizado em nosso laboratório segundo o procedimento descrito por Nalin e colaboradores. ${ }^{10} \mathrm{O}$ sistema vítreo estudado foi o $1-\mathrm{x}\left(50 \mathrm{WO}_{3}\right.$ $-30 \mathrm{SbPO}_{4}-20 \mathrm{PbO}$ )-xAgCl (sendo que 0 x 25 , em \% molar). As 
massas das composições vítreas contendo diferentes concentrações de $\mathrm{AgCl}$ foram pesadas e, posteriormente, homogeneizadas com o auxílio de um almofariz de ágata. Em seguida, a mistura de pós foi transferida para um cadinho de platina e levado ao forno para a fusão. Os vidros foram fundidos a $1100{ }^{\circ} \mathrm{C}$ durante $30 \mathrm{~min}$. Após esse período, a temperatura do forno foi reduzida para $1000{ }^{\circ} \mathrm{C}$, o líquido foi deixado nesta temperatura durante $5 \mathrm{~min}$ e em seguida foi vertido em um molde de aço inoxidável pré-aquecido a $20{ }^{\circ} \mathrm{C}$ abaixo da temperatura de transição vítrea, Tg. Esse procedimento é crítico para o sistema em estudo uma vez que vertendo o líquido na temperatura de $1100{ }^{\circ} \mathrm{C}$ se favorece um processo de decomposição/redução da prata, resultando no escurecimento do vidro. Os monolitos vítreos foram mantidos $20^{\circ} \mathrm{C}$ abaixo da $\mathrm{Tg}$ por $2 \mathrm{~h}$ para recozimento. Este procedimento serve para eliminar as tensões residuais internas do vidro, decorrentes do resfriamento rápido do líquido e visa aumentar a resistência mecânica do mesmo.

O acabamento final das amostras foi obtido através do polimento dos vidros com lixas de $\mathrm{SiC}$ com diferentes granulometrias (800, 1200,2400 e 4000 mesh).

\section{Crescimento das nanopartículas}

Nanopartículas foram obtidas por meio do tratamento térmico dos monolitos vítreos em temperaturas determinadas a partir de um estudo sistemático baseado nos dados de análise térmica de cada amostra. Para induzir o crescimento controlado das nanopartículas nos vidros, a temperatura de tratamento usada foi $50{ }^{\circ} \mathrm{C}$ acima da temperatura de transição vítrea.

\section{Caracterização dos vidros}

As curvas de calorimetria exploratória diferencial (DSC) foram obtidas utilizando um equipamento 2910 da TA Instrumentos. As amostras vítreas foram moídas em almofariz de ágata, colocadas em cadinhos de alumínio e aquecidas com velocidade de $10{ }^{\circ} \mathrm{C} \mathrm{min}^{-1}$, em atmosfera de $\mathrm{N}_{2}$, a partir da temperatura ambiente até $600^{\circ} \mathrm{C}$. As curvas de DSC permitem obter as temperaturas características dos vidros; transição vítrea, $\mathrm{Tg}$, temperatura do início da cristalização, Tx, e temperatura do máximo da cristalização, Tp. O erro estimado na determinação das temperaturas características é de $\pm 2{ }^{\circ} \mathrm{C}$ para Tg e Tx e $1{ }^{\circ} \mathrm{C}$ para Tp.

As amostras foram caracterizadas por difração de raios X, no intervalo de $2 \theta$ entre 4 e 70 graus tanto para a comprovação da presença da fase não cristalina, como para identificação de fases cristalinas.

Os espectros na região do infravermelho foram obtidos dos vidros moídos e dispersos em pastilhas de $\mathrm{KBr}$ usando um equipamento Perkin Elmer FT-IR Spectrometer Spectrum, 2000 no intervalo de comprimento de onda de $400-4000 \mathrm{~cm}^{-1}$.

Os espectros de espalhamento Raman foram obtidos usando um equipamento LabRAM da Jobin-Yvon com comprimento de onda de $632 \mathrm{~nm}$ e feixe de $5 \mu \mathrm{m}$ de diâmetro (Micro Raman).

Os espectros na região do ultravioleta-visível-infravermelho próximo foram obtidos usando um equipamento Cary 5000 da Varian. A região estudada se estende de 2000 a $350 \mathrm{~nm}$ com resolução de $4 \mathrm{~nm}$.

Os índices de refração foram obtidos usando espectroscopia M-Lines em um instrumento Metricon 2010 em três comprimentos de onda, 543, 632 e $1550 \mathrm{~nm}$, no modo TE. Os erros estimados para os valores de índice de refração são de $10^{-4}$.

A identificação da morfologia das nanopartículas foi feita usando um microscópio eletrônico de transmissão Phillips CM 120. A microscopia eletrônica de alta resolução foi realizada utilizando um microscópio Jeol, modelo JEM 2010 ARP. A preparação da amostra foi feita primeiramente, moendo-se um pedaço do vidro em um almofariz. O pó foi suspenso em etanol utilizando um banho ultrassônico e, posteriormente, gotas da suspensão foram depositadas sobre uma grade de cobre recoberta com filme de carbono.

A composição química aproximada dos vidros foi obtida por espectrometria de dispersão de energia de raios X (EDS) usando também o equipamento Jeol, modelo JEM 2010 ARP.

Para as medidas eletroquímicas uma célula eletroquímica foi adaptada e acoplada a um microscópio, permitindo observar in situ possíveis mudanças de coloração do vidro aderido à superfície do eletrodo de trabalho. ${ }^{11}$ Como eletrodo auxiliar foram utilizados dois fios de platina curto-circuitados e como eletrodo referência Ag/ $\mathrm{AgCl} / \mathrm{KCl}_{\text {sat }}$ colocado em capilar de Luggin. O eletrólito de suporte utilizado foi $\mathrm{Na}_{2} \mathrm{SO}_{4} 0,2 \mathrm{~mol} \mathrm{~L}^{-1} \mathrm{em} \mathrm{pH} 2$. O eletrodo de trabalho foi um eletrodo de pasta de carbono ${ }^{11}$ (CPE) modificado, onde o pó do material de estudo foi aderido à superfície do eletrodo (eletrodo tipo carimbo). Esse eletrodo de trabalho foi então centralizado na célula de teflon e coberto pelo eletrólito suporte. O equipamento utilizado para as medidas eletroquímicas foi um bipotenciostato da Pine, modelo AFRDE 4, acoplado a um microscópio da Quimis, modelo Q734ZT e a uma câmera digital colorida da Quimis, modelo SDC-312. ${ }^{11}$ Para as medidas foi utilizado o programa de perturbação de potenciais de $1,0 \rightarrow-1,0 \rightarrow 1,0 / \mathrm{V} / \mathrm{Ag} / \mathrm{AgCl} / \mathrm{KCl}_{\text {sat, }}$ exceto quando outros intervalos de potencial são indicados.

\section{RESULTADOS E DISCUSSÃO}

\section{Caracterização térmica, estrutural e óptica dos vidros}

As amostras vítreas do sistema $1-\mathrm{x}\left(0,5 \mathrm{WO}_{3}-0,3 \mathrm{SbPO}_{4}-0,2 \mathrm{PbO}\right)$ $\mathrm{xAgCl}$ com $(0 \leq \mathrm{x} \leq 25 \mathrm{em} \%$ molar $)$ foram estudadas por análise térmica (DSC) para determinação das temperaturas características. Essas composições foram escolhidas visando principalmente a obtenção de vidros contendo altos valores de índice de refração e que permitissem a cristalização homogênea das nanopartículas de prata por tratamento térmico. As curvas de DSC são mostradas na Figura 1, enquanto que a Tabela 1 resume as concentrações estudadas, as temperaturas características dos vidros e o parâmetro de estabilidade ( $\mathrm{Tx}-\mathrm{Tg}$ ) obtido para cada composição.

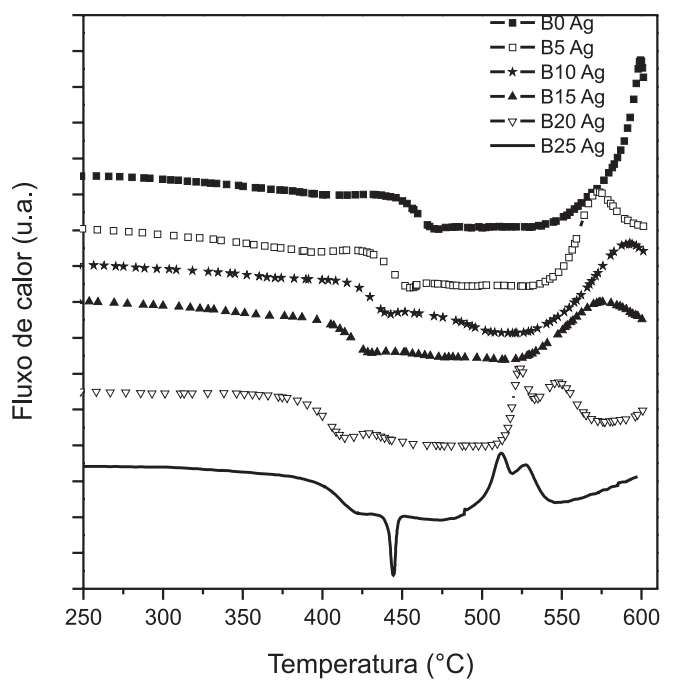

Figura 1. Curvas de análise térmica para as amostras vítreas contendo diferentes concentrações de prata

Como pode ser observado, os valores de $\mathrm{Tg}$ diminuem com o aumento da concentração de $\mathrm{AgCl}$ até o limite de $20 \%$ em mol do cloreto, porém, para a concentração maior, a Tg permanece constante. 
Tabela 1. Composições, temperaturas características e parâmetros de estabilidade obtidos para as amostras vítreas contendo diferentes quantidades de prata

\begin{tabular}{ccccccccc}
\hline \multirow{2}{*}{ Amostra } & \multicolumn{4}{c}{ Composição* } & \multicolumn{3}{c}{ Temperaturas características } \\
\cline { 2 - 7 } & $\mathrm{WO}_{3}$ & $\mathrm{SbPO}_{4}$ & $\mathrm{PbO}$ & $\mathrm{AgCl}$ & $\mathrm{Tg}$ & $\mathrm{Tx}$ & $\mathrm{Tp}$ \\
\hline $\mathrm{B} 0 \mathrm{Ag}$ & 50 & 30 & 20 & - & 451 & 580 & 600 & 129 \\
$\mathrm{~B} 5 \mathrm{Ag}$ & 47,5 & 28,5 & 19 & 5 & 433 & 551 & 565 & 118 \\
$\mathrm{~B} 10 \mathrm{Ag}$ & 45 & 27 & 18 & 10 & 422 & 547 & 590 & 125 \\
$\mathrm{~B} 15 \mathrm{Ag}$ & 42,5 & 25,5 & 17 & 15 & 408 & 533 & 575 & 125 \\
$\mathrm{~B} 20 \mathrm{Ag}$ & 40 & 24 & 16 & 20 & 389 & 516 & 524 & 127 \\
$\mathrm{~B} 25 \mathrm{Ag}$ & 37,5 & 22,5 & 15 & 25 & 390 & 500 & 512 \\
\hline
\end{tabular}

* Composição teórica (deve-se levar em consideração o fator de correção pelo fato da liberação de $\mathrm{Cl}_{2}$ durante o processo de preparação dos vidros e consequente formação de $\mathrm{Ag}^{0}$ ).

Da mesma maneira, o parâmetro de estabilidade, Tx - Tg se mantém praticamente constante até a amostra B20Ag (Tabela 1) e diminui para a amostra contendo $25 \%$ em mol de AgCl. No DSC da amostra $\mathrm{B} 25 \mathrm{Ag}$ nota-se o aparecimento de um pico endotérmico, em aproximadamente $445{ }^{\circ} \mathrm{C}$, indicando a fusão de uma fase cristalina, heterogênea à composição vítrea. De fato, a temperatura de fusão do $\mathrm{AgCl}$ cristalino é de $445^{\circ} \mathrm{C} .{ }^{12}$

Os dados de difração de raios $\mathrm{X}$ comprovam a presença da fase cristalina cúbica referente ao mineral clorargirita $(\mathrm{AgCl})$, como pode ser observado na Figura $2 .{ }^{13}$ Entretanto, vale ressaltar que a amostra B25Ag se trata de uma vitrocerâmica, uma vez que foi observada a presença da transição vítrea por DSC. ${ }^{14} \mathrm{Na}$ Figura 2 também são mostrados os difratogramas para as demais composições comprovando a formação da fase não cristalina.

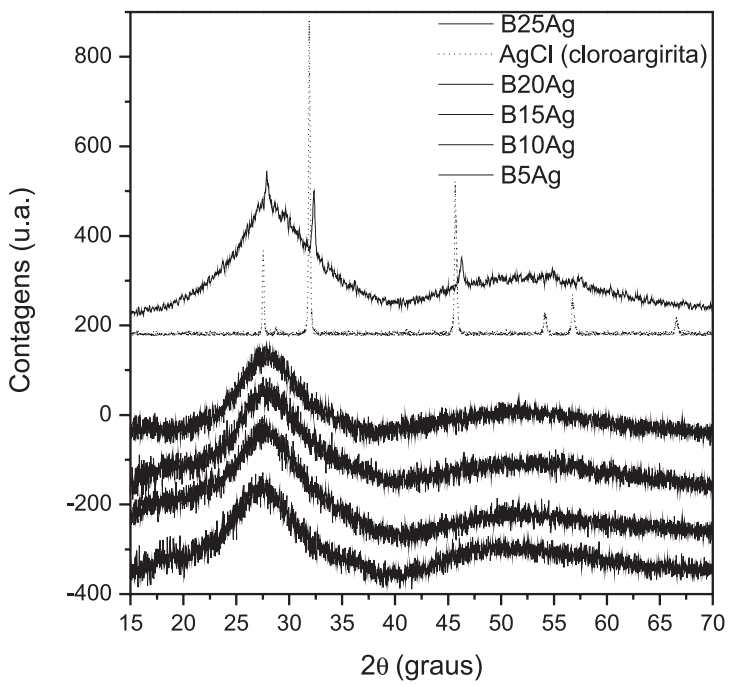

Figura 2. Difração de raios $X$ da amostra B25Ag comprovando a formação de uma vitrocerâmica e a comparação com a fase cristalina de $\mathrm{AgCl}$

A espectroscopia na região do infravermelho visa analisar possíveis variações nas energias de ligação causadas pelas distorções das unidades estruturais que compõem o vidro após a adição do $\mathrm{AgCl}$. As curvas obtidas podem ser observadas na Figura 1S, material suplementar. As atribuições das bandas foram feitas com base nos espectros dos compostos cristalinos $\mathrm{WO}_{3}$ e $\mathrm{SbPO}_{4}$ e já foram bem discutidas anteriormente. ${ }^{15}$ Os espectros na região do infravermelho médio do $\mathrm{PbO}$ e do $\mathrm{AgCl}$ não foram relacionados, uma vez que as vibrações do $\mathrm{Pb}-\mathrm{O}$ ocorrem em $505 \mathrm{~cm}^{-1}$ e são de baixa intensidade e, provavelmente, estão encobertas pelas vibrações do $\mathrm{SbO}_{3}$ e $\mathrm{PO}_{4}$ que ocorrem em 593 e $473 \mathrm{~cm}^{-1}$, respectivamente. $\mathrm{O} \mathrm{AgCl}$ é iônico e não apresenta vibrações nesta região do espectro. Como se observa na
Figura 1S, material suplementar, praticamente não há variação entre os espectros. Isto se deve, primeiramente, ao fato de que a relação entre os compostos $\mathrm{WO}_{3}, \mathrm{SbPO}_{4}$ e $\mathrm{PbO}$ não varia em decorrência do aumento da concentração de $\mathrm{AgCl}$ e como se poderia esperar, não há vibrações referentes ao cloreto de prata nesta faixa espectral. Uma possível explicação para este comportamento sugere que o cloreto de prata entraria na estrutura vítrea na forma iônica, isto é, como $\mathrm{Ag}^{+} \mathrm{e}$ $\mathrm{Cl}^{-}$, ocupando os interstícios da matriz, sem que houvesse ligações químicas com os outros componentes que formam o vidro. Entretanto, outra hipótese deve ser considerada e necessita de uma discussão um pouco mais profunda. Esta outra abordagem pode ser feita levando-se em consideração que a temperatura de fusão do $\mathrm{AgCl}$ é da ordem de $445{ }^{\circ} \mathrm{C}$ e que a temperatura de fusão do vidro é de $1100{ }^{\circ} \mathrm{C}$. Assim, se poderia esperar a decomposição do $\mathrm{AgCl}$, e a consequente perda de cloreto durante o processo de fusão segundo a reação 1 :

$$
2 \mathrm{AgCl}_{(s)} \underset{\Delta}{\longrightarrow} 2 \mathrm{Ag}_{(s)}^{0}+\mathrm{Cl}_{2(g)}
$$

Neste cenário temos que admitir que o vidro, logo após ser preparado por choque térmico, já possui prata metálica. Entretanto, em ambos os casos, seja a formação do par $\mathrm{Ag}^{+} / \mathrm{Cl}^{-}$ou a formação de $\mathrm{Ag}^{0}$, seria muito difícil visualizar alguma variação nos espectros de infravermelho devido ao par iônico e ao diminuto tamanho das partículas, respectivamente.

Apesar dos vidros apresentarem bandas mais largas que nos cristais, devido à desordem estrutural, se observa que a adição de diferentes concentrações de $\mathrm{AgCl}$ influencia nas energias de ligação dos grupos fosfatos, uma vez que ocorre um pequeno deslocamento para menores energias, tanto das bandas referentes aos estiramentos simétrico e assimétrico (1054 e $934 \mathrm{~cm}^{-1}$, respectivamente) do grupo $\mathrm{PO}_{4}$, como da deformação simétrica $\left(628 \mathrm{~cm}^{-1}\right)$, indicando uma diminuição da constante de força da ligação P-O. As bandas referentes às vibrações do octaedro $\mathrm{WO}_{6}$ e do poliedro $\mathrm{SbO}_{3}$ permanecem inalteradas, mesmo após a adição de $25 \%$ em mol de $\mathrm{AgCl}$.

Na Figura 2S, material suplementar, são mostrados os espectros Raman obtidos para os vidros. As atribuições das bandas foram feitas em relação aos compostos cristalinos de referência e já foram bem discutidas por Nalin e colaboradores. ${ }^{15}$ Como se pode observar, os espectros Raman apresentam duas bandas largas, uma de baixa (300-400 $\left.\mathrm{cm}^{-1}\right)$ e outra de maior energia $\left(600-1000 \mathrm{~cm}^{-1}\right)$. A banda de alta energia apresenta uma absorção principal na região de 909 $\mathrm{cm}^{-1}$ e outra em, aproximadamente, $820 \mathrm{~cm}^{-1}$. A primeira absorção é atribuída à formação de ligações do tipo $\mathrm{W}-\mathrm{O}_{\mathrm{t}}$, enquanto que a segunda corresponde ao estiramento simétrico da vibração W-O-W. ${ }^{16}$ Assim, como observado nos espectros na região do infravermelho, os espectros de deslocamento Raman para os vidros não apresentam variações na posição das vibrações e nem alterações da intensidade das bandas, o que reforça a explicação dada para o infravermelho de 
que a adição do cloreto de prata não influencia na estrutura da matriz. $\mathrm{O} \mathrm{AgCl}$ cristalino apresenta algumas bandas no Raman, entretanto, nenhuma delas é evidente no espectro da amostra B25Ag. ${ }^{13}$ Isto reforça a possibilidade das partículas estarem em escala nanométrica e, portanto, serem de difícil detecção, considerando-se a resolução do Raman.

Quanto às propriedades ópticas dos vidros, os monolitos apresentam colorações diferentes dependendo da concentração de prata. As amostras contendo até $15 \% \mathrm{em} \mathrm{mol} \mathrm{de} \mathrm{AgCl}$ são amarelas enquanto que as amostras com concentrações superiores apresentaram coloração vermelha. Estas características são confirmadas pelos espectros de absorção na região do UV-Vis, como mostrado na Figura 3.

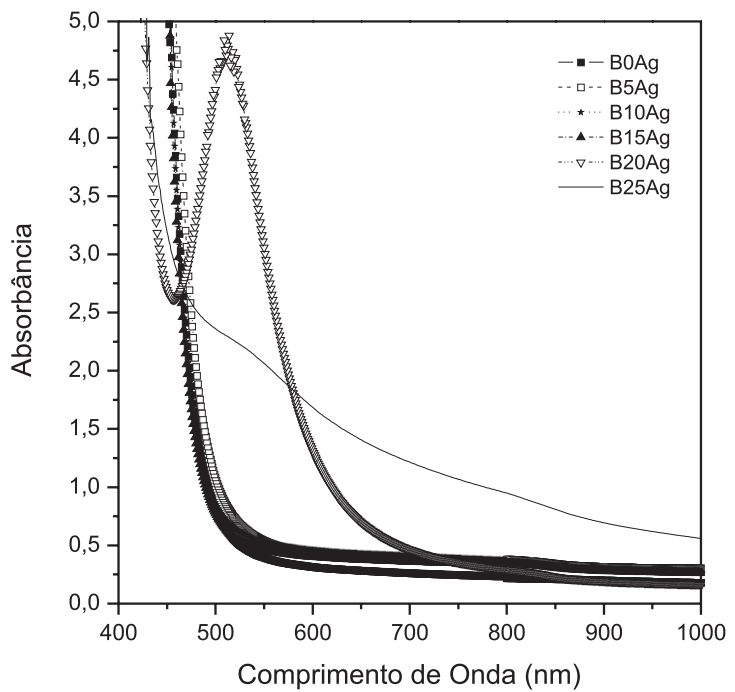

Figura 3. Espectros de absorção na região do visível e infravermelho próximo obtido dos monólitos vítreos polidos. Os espectros das amostras B20Ag e B25Ag foram obtidos de lâminas de vidros com espessura inferior a 0,5 mm, devido à intensa absorção das amostras nesta região

Nota-se que as amostras B20Ag e B25Ag apresentam uma banda de absorção na região de $515 \mathrm{~nm}$. Estes resultados são de grande importância e também corroboram no sentido da formação das nanopartículas de prata. A banda de absorção, neste caso, pode ser atribuída à ressonância de plasmons superficiais (SPR), devido à presença de nanopartículas de prata metálica. ${ }^{17}$ Devemos salientar que para o aparecimento de bandas do tipo SPR é imperativo que as nanopartículas sejam metálicas. ${ }^{17}$ Dados recentes da literatura sobre vidros silicatos, ${ }^{18,19}$ alumino-silicatos, ${ }^{20}$ alumino-fosfatos ${ }^{21} \mathrm{e}$ antimonatos ${ }^{8}$ contendo prata, em escala nanométrica, mostram que a presença das nanopartículas de prata metálica apresentam a banda SPR na região do visível e suas energias podem variar desde 408 até $598 \mathrm{~nm}$. A posição da banda SPR é influenciada por vários fatores, sendo os principais: o índice de refração, $\mathrm{n}_{\mathrm{D}}$, do meio dielétrico, o tamanho das nanopartículas e sua geometria. $\mathrm{O}$ aumento do índice de refração do dielétrico, o aumento do tamanho e a irregularidade na geometria das nanopartículas deslocam a posição da banda SPR para energias menores. Neste sentido, os resultados da literatura mostram que vidros com baixo índice de refração $\left(n_{D} \sim\right.$ 1,5) apresentam SPR na região de 408 a $440 \mathrm{~nm}$, enquanto que vidros antimonetos $\left(\mathrm{n}_{\mathrm{D}} \sim 1,8\right)$ apresentam SPR na região de $545-598 \mathrm{~nm}$, embora, neste caso a irregularidade de geometria das nanopartículas também tenha contribuído para um maior deslocamento da posição da SPR para menores energias.

Portanto, com base nos resultados mostrados nas Figuras 1 a 3 e $1 \mathrm{~S}$ e $2 \mathrm{~S}$, material suplementar, considerando a reação de decomposição (1) podemos sugerir três situações: na primeira, para amostras contendo até $15 \%$ em mol de $\mathrm{AgCl}$, o tempo de fusão empregado para a preparação dos vidros é suficiente para que a reação (1) ocorra em sua totalidade. Na segunda, para amostras mais concentradas em $\mathrm{AgCl}$, o tempo de fusão usado não foi suficiente para eliminar todo o cloro e parte do $\mathrm{AgCl}$ permanece na amostra e, embora, isto não influencie nos espectros vibracionais, a alta concentração de $\mathrm{AgCl}$ convertida em $\mathrm{Ag}^{0}$ favorece o crescimento das nanopartículas, mesmo durante o processo de fusão. Por último, para a amostra B25Ag a concentração de $\mathrm{AgCl}$ é muito elevada e não pode ser totalmente incorporada à matriz vítrea, deixando parte do $\mathrm{AgCl}$ cristalino como mostrado nas Figuras $1 \mathrm{~S}$ e $2 \mathrm{~S}$, material suplementar, e também levando à formação de nanopartículas (Figura 3).

A espectroscopia M-lines foi usada para medir o $n_{D}$ dos vidros em três comprimentos de onda distintos, 543, 632 e $1550 \mathrm{~nm}$, e analisar a influência da concentração de $\mathrm{AgCl}$ na matriz. Na Figura 3S, material suplementar, é mostrada a variação do índice de refração para as diferentes composições vítreas, onde se pode observar que o aumento da concentração de $\mathrm{AgCl}$ aumenta o $\mathrm{n}_{\mathrm{D}}$ dos vidros a partir da amostra B0Ag $(2,0933)$ até a amostra B20Ag $(2,1118)$ em, aproximadamente, $2 \%$. Estes valores de $\mathrm{n}_{\mathrm{D}}$ são bastante elevados e sugerem que estes vidros possuem um grande potencial para aplicações em fotônica. Estudos baseados em óptica não linear estão atualmente em andamento para se avaliar tal potencial.

\section{Preparação de vitrocerâmicas transparentes}

A temperatura de tratamento térmico para o crescimento das nanopartículas foi determinada a partir dos dados de análise térmica. Um fato importante que deve ser relatado é que o tratamento térmico realizado em temperaturas abaixo da $\mathrm{Tg}$ não produziu qualquer variação nas propriedades ópticas, tampouco nas propriedades estruturais dos vidros; tais propriedades foram verificadas por espectroscopia na região do UV-Vis e Raman, respectivamente. Por outro lado, o tratamento térmico controlado, em temperaturas acima da $\mathrm{Tg}$, leva à formação de nanopartículas, uma vez que, nestas temperaturas a viscosidade do vidro diminui consideravelmente, permitindo a difusão dos átomos de prata. ${ }^{14}$ Considerando-se a Tg do vidro, foi escolhida a amostra B10Ag e a temperatura de $470{ }^{\circ} \mathrm{C}$ para o tratamento. Observa-se que um tratamento de 15 min nesta temperatura é suficiente para causar variação da cor da amostra, sendo que o vidro sem tratamento possui cor amarela e após o tratamento térmico passa a vermelho. A mesma amostra foi submetida a tratamentos térmicos por diferentes períodos e os espectros de absorção foram obtidos após cada tratamento, totalizando $240 \mathrm{~min}$. A mudança de cor foi estudada por espectroscopia na região do UV-Vis e observa-se que a amostra tratada é caracterizada pelo aparecimento de uma banda de absorção em aproximadamente $515 \mathrm{~nm}$, Figura 4.

A amostra tratada durante 240 min foi submetida à análise de microscopia eletrônica de transmissão, confirmando-se a presença das nanopartículas, Figura 5.

Como pode ser observado na Figura 5, a distribuição de tamanho das nanopartículas tem um máximo próximo de $6 \mathrm{~nm}$. Dessa maneira, pode-se afirmar que o tratamento térmico controlado leva à formação de nanopartículas metálicas de prata no vidro e a banda de absorção na região de $515 \mathrm{~nm}$ se trata da banda de ressonância de plasmons superficiais, devido à presença das nanopartículas de prata metálica. No inserto da Figura 5 é mostrada uma ampliação da nanopartícula, obtida por microscopia eletrônica de transmissão de alta resolução, onde é possível verificar a presença de planos cristalinos bem definidos.

$\mathrm{Na}$ Figura 4S, material suplementar, são mostrados os espectros de análise química (EDS) obtidos em dois pontos distintos da amostra. O ponto 1 se refere a uma posição sobre a nanopartícula, enquanto que o ponto 2 está situado onde, aparentemente, não há formação das 


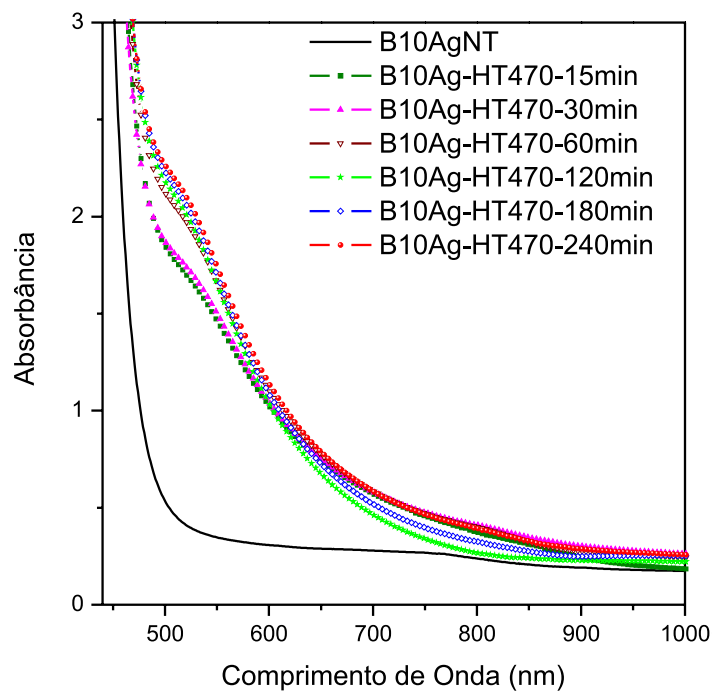

Figura 4. Espectros de absorção na região do ultravioleta e visível da amostra B10Ag tratada termicamente a $470{ }^{\circ} \mathrm{C}$ durante diferentes tempos, evidenciando a formação da banda de ressonância de plasmons em aproximadamente $515 \mathrm{~nm}$

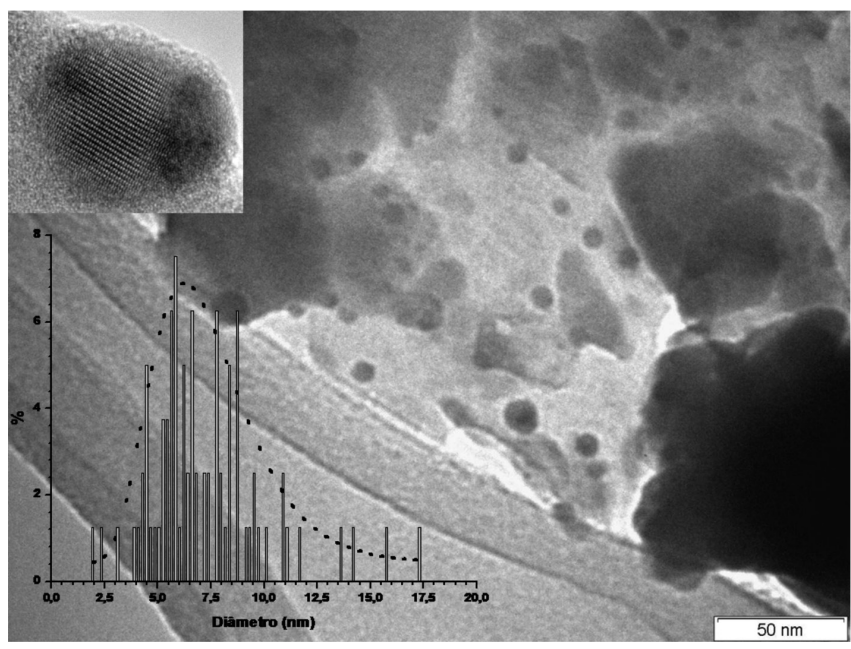

Figura 5. Micrografia obtida por microscopia eletrônica de transmissão da amostra B10Ag tratada a $470{ }^{\circ} \mathrm{C}$ por 240 min. Inserto: Distribuição média do tamanho das nanopartículas obtida pela análise de microscopia. Inserto: Micrografia obtida por microscopia eletrônica de alta resolução da amostra B10Ag tratada termicamente a $470^{\circ} \mathrm{C}$ por 240 min evidenciando a formação de planos cristalinos

nanopartículas. Como se pode notar, a diferença mais marcante está relacionada ao aumento da concentração de prata no ponto 1 . Outro fato importante e que corrobora para a hipótese de formação de prata é a ausência de $\mathrm{Cl}$ nos espectros de EDS. Os resultados de EDS, em massa e \% atômica, bem como as porcentagens de erro associadas, estão mais bem detalhados na Tabela 2 .

Como podemos comparar na Figura 5S, material suplementar, as energias da banda de absorção da amostra B20Ag e da amostra
B10Ag tratada por 240 min a $470{ }^{\circ} \mathrm{C}$ são praticamente idênticas. Este fato sugere que haja formação das nanopartículas durante o processo de preparação dos vidros. Assim, deve-se ponderar que a formação de agregados de átomos metálicos de prata, suficientemente grandes e que possibilitem a interação com a radiação eletromagnética, originando a banda de plasmon nos vidros sem tratamento térmico, deve ser dependente da concentração de $\mathrm{AgCl}$ adicionada inicialmente, como sugerido anteriormente para os vidros contendo 20 e $25 \%$ em mol de $\mathrm{AgCl}$.

A observação das nanopartículas e a cristalização homogênea dos vidros oferecem uma grande oportunidade para o estudo de propriedades físicas, tais como a variação do índice de refração não linear e a transferência de energia entre as nanopartículas e íons de terras raras. ${ }^{6,7}$ Estes dois efeitos podem ser combinados para a preparação de fibras ópticas ou guias de onda dopados com terras raras $\left(\mathrm{Tm}^{3+} \mathrm{e} / \mathrm{ou} \mathrm{Y}^{3+}\right),{ }^{22}$ com emissão na região do infravermelho médio visando a obtenção de amplificadores de banda larga com aplicações em telecomunicações. Estas possibilidades estão atualmente sendo investigadas.

\section{Propriedades eletroquímicas}

Na Figura 5S, material suplementar, são mostrados os voltamogramas obtidos para o óxido de tungstênio e vidros B0Ag, B10Ag e B25Ag. No voltamograma referente ao óxido pode-se observar um aumento da corrente negativa por volta de $0,2 \mathrm{~V} /\left(\mathrm{Ag} / \mathrm{AgCl} / \mathrm{KCl}_{\text {sat. }}\right) \mathrm{e}$ a presença de três picos de corrente catódica: ao redor de 0,$0 ;-0,25$ e $-0,73 \mathrm{~V} /\left(\mathrm{Ag} / \mathrm{AgCl} / \mathrm{KCl}_{\text {sat. }}\right)$. Além disso, há um pequeno pico de corrente catódica ao redor de $-0,5 \mathrm{~V} /\left(\mathrm{Ag} / \mathrm{AgCl} / \mathrm{KCl}_{\text {sat. }}\right)$.

Processos similares de redução também foram observados nas amostras de vidro B0Ag, B10Ag, B25Ag, onde ao redor de $0,0 \mathrm{~V} /(\mathrm{Ag} /$ $\mathrm{AgCl} / \mathrm{KCl}_{\text {sat. }}$ ) ocorre um aumento da corrente negativa, com um evento discreto de redução, enquanto um pico largo de redução é observado ao redor de $-0,25 \mathrm{e}-0,5 \mathrm{~V} /\left(\mathrm{Ag} / \mathrm{AgCl} / \mathrm{KCl}_{\text {sat. }}\right)$ para as amostras contendo $\mathrm{Ag}$ e, em $-0,8 \mathrm{~V} /\left(\mathrm{Ag} / \mathrm{AgCl} / \mathrm{KCl}_{\text {sat. }}\right)$ para a amostra sem adição de prata. A cada pico de corrente catódica há um pico correspondente de corrente anódica, respectivamente em $+0,19$; - 0.05 e -0,45 V/ $\left(\mathrm{Ag} / \mathrm{AgCl} / \mathrm{KCl}_{\text {sat. }}\right.$ ). Porém, esses picos possuem menor intensidade que os picos catódicos.

Entre $-0,25$ e $-0,8 \mathrm{~V} /\left(\mathrm{Ag} / \mathrm{AgCl} / \mathrm{KCl}_{\text {sat. }}\right)$ observamos picos de corrente catódica, característicos da redução do $\mathrm{WO}_{3}$ associado ao fenômeno eletrocrômico apresentado pelo tungstênio. ${ }^{23,24}$ Materiais contendo tungstênio são classificados como materiais eletrocrômicos catódicos e são condutores mistos, isto é, condutores iônicos e eletrônicos, dentro dos quais íons podem ser inseridos de maneira rápida e reversível. ${ }^{23-29}$ As reações eletrocrômicas do $\mathrm{WO}_{3}$ têm sido descritas por vários modelos, mas, o mais amplamente aceito é o modelo da dupla injeção, o qual assume que o processo de coloração é devido à inserção de íons monovalentes na matriz, de acordo com a Equação 2:

$$
W_{3}+x M^{+}+e^{-} \rightleftarrows M x W O_{3}
$$

Para garantir a eletroneutralidade do meio, a inserção de um íon dentro da rede do composto, em geral um cátion monovalente $\mathrm{M}^{+}$, deve ser acompanhada pela injeção de um elétron, balanceando a estrutura eletrônica do composto, de acordo com a Equação geral (2). $\mathrm{O}$ alargamento desse pico permite sugerir que nesses potenciais

Tabela 2. Resultados da microanálise química obtida por EDS em porcentagem em atômica para dois pontos distintos da amostra B10Ag tratada termicamente a $450{ }^{\circ} \mathrm{C}$ durante $240 \mathrm{~min}$

\begin{tabular}{ccccccccccc}
\hline & \multicolumn{10}{c}{ \% atômica } \\
\cline { 2 - 11 } & O-K & \%erro & P-K & \%erro & Ag-L & \%erro & Sb-L & \%erro & W-L & \%erro \\
\hline pt1 & 44,7 & 5,5 & 15,2 & 1,9 & 16,1 & 1,0 & 5,6 & 0,5 & 18,1 & 1,7 \\
pt2 & 49,3 & 5,4 & 17,2 & 1,9 & 6,9 & 1,0 & 6,1 & 0,5 & 19,2 & 1,5 \\
\hline
\end{tabular}


pode ocorrer também a redução da $\mathrm{Ag}$ e $\mathrm{Sb}$ presentes no vidro. $\mathrm{Na}$ amostra sem adição de prata observa-se a diminuição na corrente catódica, o que sugere um processo de redução menos acentuado. Invertendo-se o sentido da varredura de potencial observam-se três picos de corrente anódica para as amostras com adição de $\mathrm{Ag}$ ao redor de $-0,45 \mathrm{~V} /\left(\mathrm{Ag} / \mathrm{AgCl} / \mathrm{KCl}_{\text {sat }}\right)$, zero $\mathrm{V} /\left(\mathrm{Ag} / \mathrm{AgCl} / \mathrm{KCl}_{\text {sat. }}\right)$ e 0,1 $\mathrm{V} /\left(\mathrm{Ag} / \mathrm{AgCl} / \mathrm{KCl}_{\text {sat. }}\right)$, este último ausente nas amostras sem adição de prata, sendo, possivelmente, devido à oxidação da prata reduzida na varredura direta.

A Figura 6 mostra os perfis I-E para o vidro sem prata, obtidos com diferentes intervalos de potencial.

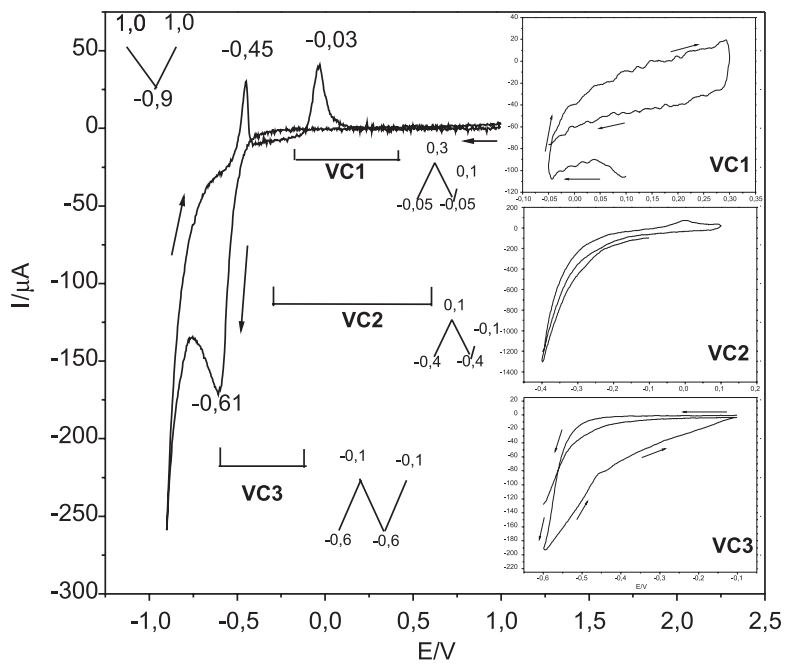

Figura 6. Voltamograma cíclico da pasta de grafite modificada com o vidro BOAg, em $\mathrm{Na}_{2} \mathrm{SO}_{4}, \mathrm{pH}=2$ e $\mathrm{v}=20 \mathrm{mV} \mathrm{s}{ }^{-1}$, com perturbação de potenciais $1.0 \mathrm{~V} \rightarrow-0,9 \mathrm{~V} \rightarrow 1,0 \mathrm{~V}$, indicando por barras as regióes de potencial que foram exploradas nos voltamogramas VC1 (com perturbação de potenciais $0,1 \mathrm{~V} \rightarrow-0,05 \mathrm{~V} \rightarrow 0,3 \mathrm{~V} \rightarrow 0,1 \mathrm{~V}$ ), VC2 (com perturbação de potenciais $-0,1 \mathrm{~V} \rightarrow-0,4 \mathrm{~V} \rightarrow 0,1 \mathrm{~V} \rightarrow-0,4 \mathrm{~V})$ e $\mathrm{VC} 3$ (com perturbação de potenciais $-0,1 \mathrm{~V} \rightarrow-0,6 \mathrm{~V} \rightarrow-0,1 \mathrm{~V} \rightarrow-0,6 \mathrm{~V})$

$\mathrm{O}$ voltamograma cíclico obtido no intervalo de $+1,0$ a $-0,9 \mathrm{~V} /(\mathrm{Ag} /$ $\mathrm{AgCl} / \mathrm{KCl}_{\text {sat. }}$ ) mostra os seguintes picos de corrente: (a) na direção de potenciais negativos observa-se um pico em - $-0,61 \mathrm{~V}$ e uma corrente de descarga de hidrogênio a partir de ca. $-0,8 \mathrm{~V}$; no sentido de potenciais positivos observam-se picos de corrente em - 0,45 e - 0,03 $\mathrm{V} /\left(\mathrm{Ag} / \mathrm{AgCl} / \mathrm{KCl}_{\text {sat. }}\right)$. Na varredura de potencial entre $-0,05$ e $+0,5$ $\mathrm{V} /\left(\mathrm{Ag} / \mathrm{AgCl} / \mathrm{KCl}_{\text {sat }}\right)$ (inserto 1 ) não foi observado qualquer pico de corrente bem definido, indicando que o pico em - $0,03 \mathrm{~V} /(\mathrm{Ag} / \mathrm{AgCl} /$ $\mathrm{KCl}_{\text {sat. }}$ ) tem origem em outro intervalo de potencial. Entre $0,1 \mathrm{e}-0,4$ $\mathrm{V} /\left(\mathrm{Ag} / \mathrm{AgCl} / \mathrm{KCl}_{\text {sat. }}\right.$ ) (inserto 2 ) observa-se, na varredura na direção de potenciais negativos, um aumento da corrente num potencial próximo a $-0,4 \mathrm{~V} /\left(\mathrm{Ag} / \mathrm{AgCl} / \mathrm{KCl}_{\text {sat. }}\right)$ que corresponde ao potencial onde se inicia a formação do pico de corrente obtido em -0,61 V/ $\left(\mathrm{Ag} / \mathrm{AgCl} / \mathrm{KCl}_{\text {sat. }}\right)$. Após a inversão do sentido da varredura observa-se apenas um pico de corrente pouco intenso em -0,03 V/(Ag/AgCl/ $\left.\mathrm{KCl}_{\text {sat. }}\right)$, sugerindo que o pico formado em $-0,61 \mathrm{~V} /\left(\mathrm{Ag} / \mathrm{AgCl} / \mathrm{KCl}_{\text {sat. }}\right)$ na varredura catódica dá origem ao pico de corrente anódica em $-0,03 \mathrm{~V} /\left(\mathrm{Ag} / \mathrm{AgCl} / \mathrm{KCl}_{\text {sat. }}\right)$. Esses dois picos de corrente devem estar associados ao processo de intercalação de $\mathrm{H}^{+}$e remoção do próton do sistema vítreo. A corrente catódica intensa ao redor de - $0,9 \mathrm{~V} /(\mathrm{Ag} /$ $\mathrm{AgCl} / \mathrm{KCl}_{\text {sat. }}$ ) corresponde principalmente à descarga do próton e na varredura inversa há um pico de corrente anódica em - $0,45 \mathrm{~V} /(\mathrm{Ag} /$ $\mathrm{AgCl} / \mathrm{KCl}_{\text {sat. }}$ ) que pode ser devido à oxidação de hidrogênio ou do composto contendo $\mathrm{H}$. No inserto $3\left(-0,1 \mathrm{a}-0,6 \mathrm{~V} /\left(\mathrm{Ag} / \mathrm{AgCl} / \mathrm{KCl}_{\text {sat. }}\right)\right)$, observa-se um aumento de corrente catódica a partir de $-0,3 \mathrm{~V} /(\mathrm{Ag} /$ $\mathrm{AgCl} / \mathrm{KCl}_{\text {sat. }}$ ) e na varredura inversa a corrente continua catódica e maior em módulo do que a observada na varredura direta, sugerindo um processo de nucleação (ou intercalação).

A Figura 7 mostra os voltamogramas cíclicos obtidos para a amostra que contém $20 \%$ molar em prata em diferentes intervalos de potencial. Os picos de corrente assinalados para o eletrodo de CPE modificado com o vidro sem a presença de prata apresentam-se nos mesmos potenciais do eletrodo contendo prata, porém, o pico de corrente catódica em $-0,61 \mathrm{~V} /\left(\mathrm{Ag} / \mathrm{AgCl} / \mathrm{KCl}_{\text {sat. }}\right)$ se apresenta alargado e com menor intensidade do que no eletrodo sem prata. Além disso, surgiram mais dois picos de corrente em $-0,29 \mathrm{~V} /\left(\mathrm{Ag} / \mathrm{AgCl} / \mathrm{KCl}_{\text {sat. }}\right.$ ) (catódico) e em $+0,29 \mathrm{~V} /\left(\mathrm{Ag} / \mathrm{AgCl} / \mathrm{KCl}_{\text {sat. }}\right.$ ) (anódico), sugerindo que esses picos podem estar relacionados com a presença da prata no eletrodo.

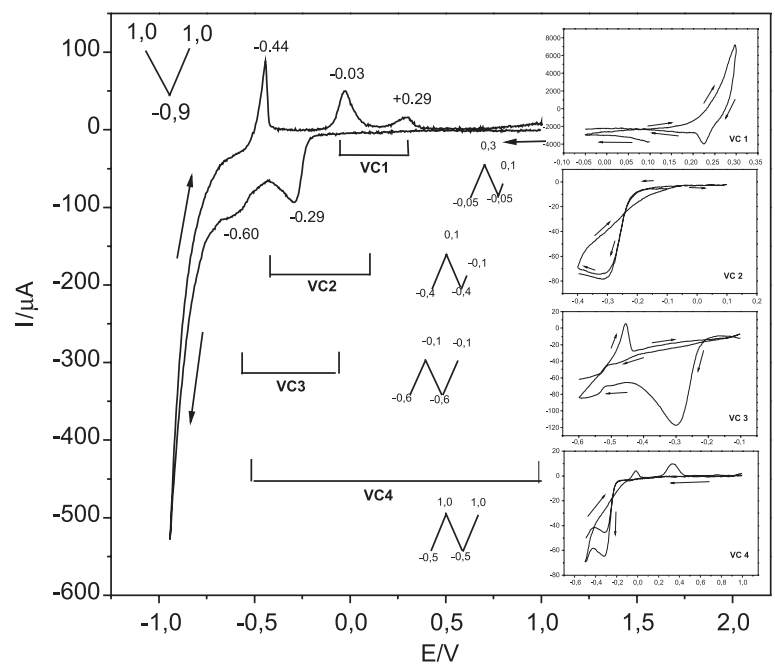

Figura 7. Voltamograma cíclico da pasta de grafite modificada com o vidro B25 $\mathrm{Ag}$, em $\mathrm{Na}_{2} \mathrm{SO}_{4}, \mathrm{pH}=2$ e $v=20 \mathrm{mV} \mathrm{s}^{-1}$, com perturbação de potenciais $1.0 \mathrm{~V} \rightarrow-0,9 \mathrm{~V} \rightarrow 1,0 \mathrm{~V}$, indicando por barras as regióes de potencial que foram exploradas nos voltamogramas VC1 (com perturbação de potenciais $0,1 \mathrm{~V} \rightarrow-0,05 \mathrm{~V} \rightarrow 0,3 \mathrm{~V} \rightarrow 0,1 \mathrm{~V}$ ), VC2 (com perturbação de potenciais $-0,1 \mathrm{~V} \rightarrow-0,4 \mathrm{~V} \rightarrow 0,1 \mathrm{~V} \rightarrow-0,4 \mathrm{~V})$, VC3(com perturbação de potenciais $-0,1 \mathrm{~V} \rightarrow-0,6 \mathrm{~V} \rightarrow-0,1 \mathrm{~V} \rightarrow-0,6 \mathrm{~V})$ e VC4(com perturbação de potenciais $-1,0 \mathrm{~V} \rightarrow-0,5 \mathrm{~V} \rightarrow 1,0 \mathrm{~V} \rightarrow-0,5 \mathrm{~V})$

Para buscar uma explicação para a presença desses picos, vários voltamogramas cíclicos foram obtidos em diferentes intervalos de potencial, conforme mostrado nos insertos da Figura 7. Verifica-se, no inserto 1 , que corresponde ao intervalo de potencial - $0,1 \mathrm{a}+0,3 \mathrm{~V} /$ $\left(\mathrm{Ag} / \mathrm{AgCl} / \mathrm{KCl}_{\text {sat }}\right)$, iniciando-se a varredura de potencial no sentido de potenciais crescentes, a presença de um pico anódico em $+0,3$ $\mathrm{V} /\left(\mathrm{Ag} / \mathrm{AgCl} / \mathrm{KCl}_{\text {sat. }}\right)$, atribuído à dissolução da prata metálica, uma vez que no voltamograma cíclico principal não há picos de redução neste intervalo de potencial. Por outro lado, ao inverter o sentido da varredura observa-se um pico de corrente catódica em $+0,22 \mathrm{~V} /(\mathrm{Ag} /$ $\left.\mathrm{AgCl} / \mathrm{KCl}_{\text {sat. }}\right)$, típico da redução de um íon metálico formado pela dissolução do metal. Esses resultados demonstram que há prata na forma metálica no vidro que compõe o eletrodo, a qual deve ter sido formada durante o processo de preparação do material vítreo.

No intervalo de potencial entre $+0,15$ e $-0,4 \mathrm{~V} /\left(\mathrm{Ag} / \mathrm{AgCl} / \mathrm{KCl}_{\text {sat }}\right)$ (inserto 2) verifica-se que há um aumento brusco da corrente catódica a partir de $-0,15 \mathrm{~V} /\left(\mathrm{Ag} / \mathrm{AgCl} / \mathrm{KCl}_{\text {sat. }}\right)$ que dá origem ao pico de corrente catódica centrado em $-0,3 \mathrm{~V} /\left(\mathrm{Ag} / \mathrm{AgCl} / \mathrm{KCl}_{\text {sat }}\right)$. Nota-se também que ao inverter o sentido da varredura em $-0,4 \mathrm{~V} /\left(\mathrm{Ag} / \mathrm{AgCl} / \mathrm{KCl}_{\text {sat. }}\right)$ há o cruzamento do registro da corrente com o da varredura direta, típico de um processo de nucleação e crescimento. Considerando que este pico e esse cruzamento não foram observados no eletrodo sem prata, sugere-se que algum composto de prata (cloreto ou óxido) pode estar se reduzindo para formar mais prata metálica. 
Outro resultado interessante é observado no inserto 3, com varredura de potencial entre $-0,1$ e $-0,6 \mathrm{~V} /\left(\mathrm{Ag} / \mathrm{AgCl} / \mathrm{KCl}_{\text {sat. }}\right)$, onde se observa a formação do pico em $-0,3 \mathrm{~V} /\left(\mathrm{Ag} / \mathrm{AgCl} / \mathrm{KCl}_{\text {sat. }}\right)$ seguido do início do pico ao redor de $-0,6 \mathrm{~V} /\left(\mathrm{Ag} / \mathrm{AgCl} / \mathrm{KCl}_{\text {sat }}\right)$ e na varredura inversa se observa o pico em $-0,45 \mathrm{~V} /\left(\mathrm{Ag} / \mathrm{AgCl} / \mathrm{KCl}_{\text {sat }}\right)$, presente também no eletrodo sem prata. Isto sugere que esse pico em $-0,45$ $\mathrm{V} /\left(\mathrm{Ag} / \mathrm{AgCl} / \mathrm{KCl}_{\text {sat. }}\right)$ corresponde à oxidação do material formado ao redor de $-0,6 \mathrm{~V} /\left(\mathrm{Ag} / \mathrm{AgCl} / \mathrm{KCl}_{\text {sat. }}\right)$ na varredura direta. Quando se inverte novamente o sentido da varredura de potencial, agora no sentido negativo de potenciais, não se observa o pico em $-0,3 \mathrm{~V} /(\mathrm{Ag} / \mathrm{AgCl} /$ $\mathrm{KCl}_{\text {sat. }}$ ) (presente na varredura direta), o que demonstra que o mesmo corresponde à redução de compostos localizados na superfície do eletrodo e que não voltam a ser formados neste intervalo de potencial.

No inserto 4, a varredura de potencial iniciou em $+1,0 \mathrm{~V} /(\mathrm{Ag} /$ $\mathrm{AgCl} / \mathrm{KCl}_{\text {sat. }}$ ) e prosseguiu até $-0,5 \mathrm{~V} /\left(\mathrm{Ag} / \mathrm{AgCl} / \mathrm{KCl}_{\text {sat. }}\right)$, quando foi invertido o sentido da mesma. Na varredura direta, observa-se o pico em $-0,3 \mathrm{~V} /\left(\mathrm{Ag} / \mathrm{AgCl} / \mathrm{KCl}_{\text {sat. }}\right)$ e o início do pico que se forma em $-0,6 \mathrm{~V} /\left(\mathrm{Ag} / \mathrm{AgCl} / \mathrm{KCl}_{\text {sat }}\right)$ e ao se inverter o sentido da varredura de potencial verificam-se os picos em $-0,03 \mathrm{~V} /\left(\mathrm{Ag} / \mathrm{AgCl} / \mathrm{KCl}_{\text {sat. }}\right)$ (menos intenso) e $+0,3 \mathrm{~V} /\left(\mathrm{Ag} / \mathrm{AgCl} / \mathrm{KCl}_{\text {sat. }}\right)$, que confirmam as atribuições feitas anteriormente.

Assim, os resultados eletroquímicos sugerem a presença de prata metálica e de algum outro composto de prata no material vítreo (provavelmente, óxido ou cloreto de prata), sendo que a prata metálica se oxida ao redor de $+0,3 \mathrm{~V} /\left(\mathrm{Ag} / \mathrm{AgCl} / \mathrm{KCl}_{\text {sat. }}\right)$ e o composto de prata se reduz ao redor de $-0,3 \mathrm{~V} /\left(\mathrm{Ag} / \mathrm{AgCl} / \mathrm{KCl}_{\text {sat. }}\right)$. A presença do composto de prata interfere na intensidade do pico em - $0,6 \mathrm{~V} /(\mathrm{Ag} / \mathrm{AgCl} /$ $\mathrm{KCl}_{\text {sat. }}$ ), provavelmente porque diminui sua quantidade na superfície que, agora, passa a ser ocupada também por composto de prata e partículas de prata metálica.

\section{CONCLUSÕES}

Foram obtidas novas matrizes vítreas estáveis frente à cristalização contendo diferentes concentrações de prata. A incorporação de $\mathrm{AgCl}$ à matriz aumenta o índice de refração dos vidros. Observa-se que para concentrações de $\mathrm{AgCl}$ de até $15 \% \mathrm{em}$ mol pode-se formar $\mathrm{Ag}^{0}$ durante o processo de fusão, entretanto, os agregados possuem tamanhos muito reduzidos. Para concentração de $20 \%$ em mol observa-se a presença de $\mathrm{Ag}^{0}$, entretanto, com a formação de nanopartículas maiores que puderam ser caracterizadas pelo aparecimento da banda de plasmon da prata na região do visível. A amostra contendo $25 \%$ em mol de $\mathrm{AgCl}$ é uma vitrocerâmica, apresentado a $\mathrm{Tg}$ e a presença de uma fase cristalina de $\mathrm{AgCl}$.

Vitrocerâmicas transparentes foram preparadas controlando-se o tempo de tratamento térmico e a presença de prata metálica foi confirmada pelo aparecimento da SPR na região do visível, por análise química e por voltametria cíclica, onde os resultados sugerem a presença de $\mathrm{Ag}^{0}$ e outra espécie de prata na forma de $\mathrm{Ag}^{+}$para a amostra contendo $25 \%$ em mol de $\mathrm{AgCl}$.

\section{MATERIAL SUPLEMENTAR}

Está disponível em http://quimicanova.sbq.org.br, na forma de arquivo PDF, com acesso livre e é constituído pelas Figuras 1S a 6S.

\section{AGRADECIMENTOS}

À FAPESP pelo auxílio financeiro. E aos Prof. Dr. Y. Messaddeq e
Dr. S. J. L. Ribeiro do LAMF (IQ-UNESP) pelas medidas de análise térmica, Raman e M-Lines e ao LME-LNLS pelas medidas usando o FEG-SEM. A. V. Benedetti agradece ao CNPq (proc. 305890/2010-7).

\section{REFERÊNCIAS}

1. Pasquazi, A.; Stivala, S.; Assanto, G.; Gonzala, J.; Solis, J.; Phys. Rev. A: At., Mol., Opt. Phys. 2008, 4, 043808.

2. Gomes, L. A.; de Araújo, C. B.; Messias, D. N.; Misoguti, L.; Zílio, S. C.; Nalin, M.; Messaddeq, Y.; J. Appl. Phys. 2006, 100, 116105.

3. Dos Santos, F. E. P.; de Araujo, C. B.; Gomes, A. S. L.; Fedus, K.; Boudebs, G.; Manzani, D.; Messaddeq, Y.; J. Appl. Phys. 2009, 106, 063507.

4. Gomez, L. A.; dos Santos, F. E. P.; Gomes, A. S. L.; de Araújo, C. B.; Kassab, L. R. P.; Hara, W. G.; J. Appl. Phys. 2008, 92, 141916.

5. Chen, F.; Daí, S.; Xu, T.; Shen, X.; Song, B.; Lin, C.; Wang, X.; Liu, C.; Xu, K.; Heo, J.; J. Opt. Soc. Am. B 2011, 28, 1283.

6. Eichelbaun, M.; Rademann, K.; Adv. Funct. Mater. 2009, 19, 2045.

7. Assunção, T. A. A.; da Silva, D. M.; Kassab, L. R. P.; Martinelli, J. R.; de Araújo, C. B.; J. Non-Cryst. Solids 2010, 356, 2465.

8. Som, T.; Karmakar, B.; J. Appl. Phys. 2009, 105, 013102.

9. Lakshminarayana, G.; Qiu, J. R. J.; J. Alloys Compd. 2009, 478, 630.

10. Nalin, M.; Messaddeq, Y.; Ribeiro, S. J. L.; Poulain, M.; Briois, V.; Brunklaus, G.; Rosenhahn, C.; Mosel, B. D.; Eckert, H., J. Mater. Chem. 2004, 14, 3398.

11. Alencar, M. A. S.; Benedetti, A. V.; Fugivara, C. S.; Messaddeq, Y.; Quim. Nova 2010, 33, 1394.

12. Lide, D. R.; CRC Handbook of Chemistry and Physics, $84^{\text {th }}$ ed., CRC Press: Boca Raton, 2003.

13. Base Ruff de dados espectroscópicos ficha nº R060959, HTTP://rruff. info/, acessada em Novembro 2010.

14. Yamane, M.; Asahara, Y.; Glasses for Photonics, $1^{\text {st }}$ ed., Cambridge University Press: Cambridge, 2000, cap. 1.

15. Nalin, M.; Poirier, G.; Ribeiro, S. J. L.; Messaddeq, Y.; Cescato, L.; J. Non-Cryst. Solids 2007, 353, 1592.

16. Charton, P.; Gengembre, L.; Armand, P.; J. Solid State Chem. 2002, 168, 175.

17. Kreibig U.; Vollmer M.; Optical properties of metal clusters, Springer Series in Materials Science 25, Springer Verlag: Berlin Heidelberg, 2010, cap. 1.

18. Sidorov, A. I.; Tsekhomskii, V. A.; Glass Phys. Chem. 2008, 34, 693.

19. Zeng, H.; Qiu, J.; Yuan, S.; Yang, Y.; Chen, G.; Ceram. Int. 2008, 34, 605.

20. Zhang, J.; Dong, W.; Sheng, J.; Zheng, J.; Li, J.; Qiao, L.; Jiang, L.; J. Cryst. Growth 2008, 310, 234.

21. Jimenez, J. A.; Lysenko, S.; Zhang, G.; Liu, H.; J. Mater. Sci. 2007, 42, 1856.

22. Ju, S.; Watekar, P. R.; Kang, S. G.; Chung, J.; Kim, C. J.; Han, W.; J. Non-Cryst. Solids 2010, 356, 2578.

23. Oliveira, S. C.; Torresi, R. M.; Córdoba de Torresi, S. I.; Quim. Nova 2000, 23, 79.

24. Bathe, S. R.; Patil, P. S. J.; J. Appl. Phys. 2007, 40, 7423.

25. Pereira, A. C.; Kubota, L. T.; Quim. Nova 2004, 27, 725.

26. Córdoba de Torresi, S. I.; Gorenstein, A.; Torresi, R. M.; Vázquez, M. V.; J. Electroanal. Chem. 1991, 318, 131.

27. Déb, S. K.; Sol. Energy Mater. Sol. Cells 2008, 92, 245.

28. Bathe, S. R.; Patil, P. S.; J. Phys. D: Appl. Phys. 2007, 40, 7423.

29. Sivakumar, R.; Gopalakrishnan, R.; Jayachandran, M.; Sanjeeviraja, C.; Smart Mater. Struct. 2006, 15, 877. 